\title{
Statistical Methods for Automatic Segmentation of Elastographic Images
}

\author{
Sergiu Nedevschi, Cosmin Pantilie, Tiberiu \\ Mariţa \\ TUCN, Computer Science Department \\ $\{$ Sergiu.Nedevschi | Cosmin.Pantilie | Tiberiu.Marita\} \\ (a)cs.utcluj.ro
}

\author{
Sorin Dudea \\ U MF Iuliu Hatieganu Cluj-Napoca \\ dudea@clicknet.ro
}

\begin{abstract}
Elastography is a new ultrasonic method for measuring tissues' elasticity. Besides many and widely acknowledged benefits, the method suffers severe limitations due to the high motion sensitivity and interoperator dependency to the point where it provides only qualitative information, not having, until now, any real quantification means. In this paper we present an automatic segmentation method for elastographic images based on statistical techniques. First a probabilistic model is built for every pixel in the image, derived by processing a video sequence instead of a single image. The built image contains the values with highest probability for each pixel. Next a DAEM (Deterministic Annealing Expectation Maximization) method is used for automatic image segmentation. Finally a numerical quantification of tissue elasticity is provided based on the segmentation.
\end{abstract}

\section{Introduction}

Elastography is an ultrasound based method which produces images of the local strain after applying small, external compressions [1]. Pathologic changes are generally associated with changes in underlying tissues' stiffness. However many disease processes, although altering the structure and mechanical properties of the affected tissue, are not accompanied by visible changes in the ultrasonographic images [1, 2]. Such changes can be detected using elastography.

The extremely high motion sensitivity of the technique, although extremely useful, induces diagnostic limitations. The lack of standardization of the magnitude, direction and frequency of the applied force by manual driving of the transducer is a source of uncertainty in the color encoding procedure, providing only qualitative information. In such conditions, the quantitative diagnostic capabilities of the method are limited [3]. For this last issue a solution is proposed in this paper.

\section{Error generation model}

The above mentioned limitations affect the measurement process and therefore the resulting elastogram. Such an example is shown in figure 1, where subsequent measurements of the same region yield slightly different results.

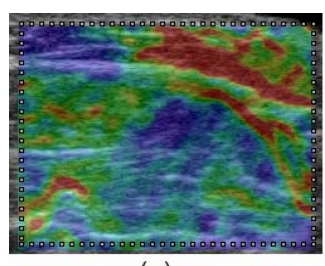

(a)

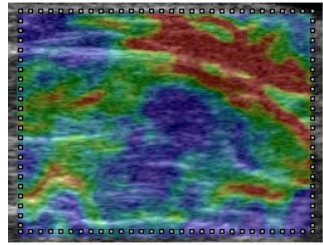

(c)

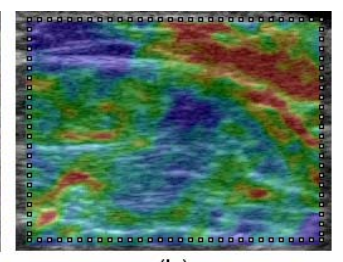

(b)

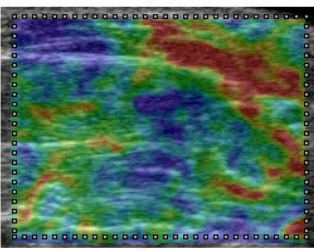

(d)
Figure 1: Subsequent measurements of the same region, by the same operator, yield different results. Images (a) to (d) are frames of a video sequence (74 fames long) of the same region.

This phenomenon is explained by measurement theory. Each time a quantity is measured the measurement is affected by errors and therefore has uncertainties. There are two types of errors which affect the measurement: measurement errors and observation errors.

Measurement errors are present due to the extremely high motion sensitivity of the device and the inherent variation introduced by manual driving of the transducer. 
Observation errors are dominated by the random error component. These errors are caused by unknown or uncontrollable factors. They are inherently present in every measurement. A significant error is introduced by physiologic motion such as heartbeat, muscle contraction, blood flow and respiration which introduce inter-frame decorrelation [4].

We concluded that a single image (a single measurement) exhibits a high degree of uncertainty, mostly because of measurement errors. Thus, using a video sequence, i.e., a sequence of measurements under the same conditions yields considerably better results. By processing a video sequence, an image containing the most probable value for each pixel can be computed. This data is expected to be closer to the true data than any individual frame.

\section{Statistical modeling and estimation of pixel values}

Most of the commercially available medical scanners also provide video sequence capture and storage besides still images. The specific information is displayed in color hues superimposed on a grayscale sonographic image. By processing a video sequence a probabilistic model is built for every pixel such that, at the end, its most probable value is assigned. This approach is possible due to the fact that elasticity is a local tissue property and its nature is reflected by a group of pixels. The dimensions of the lesions are considerably greater then the movement induced to the tissue due to the applied pressure. For this reason only a negligibly small error is introduced for not tracking the pixel's movement.

Let $V$ be a video sequence having a length of $n$ frames. Denote by $V_{i}$ the $i$-th frame in the sequence, $i=1, \ldots$, n. Denote by $V_{i j}$ the $j$-th pixel in the $i$-th frame. The appearance frequency of the hue values for corresponding pixels $V_{i j}$, in different frames, is recorded in histogram $H_{j}$, for $i=1 . . \mathrm{n}, \mathrm{j}=1 . . \mathrm{N}$. $H_{j}(k)$ reflects the number of occurrences of value $k$ (the measured hue) for the $j$-th pixel across the different frames, $k=0, \ldots, 359$. The most probable value is the most frequently encountered one and is given by

$$
V m p_{j}=\arg \max _{k} H_{j}(k)
$$

Note that if the measurement process is affected only by observation errors, a normal distribution of the errors can be assumed. In this case the mean value be the most probable value (2).

$$
V m p_{j}=\frac{1}{360} \sum_{k=0}^{359} H_{j}(k)
$$

For each pixel in the result image, the model from equation (1) is applied.

\section{Statistical method for automatic segmentation of elastographic images}

Image segmentation is in general one of the most important steps in the analysis of the patient's imaging data. An accurate segmentation is the starting point of quantitative diagnosis in the case of elastographic images. For this purpose we introduce an extension of the application domain of the DAEM (Deterministic Annealing Expectation Maximization) algorithm [5, 6, 7,8 ] for elastographic images. The method assumes a Gaussian Mixture Model (GMM) for the characterization of the hue values distribution observed from the elastographic images.

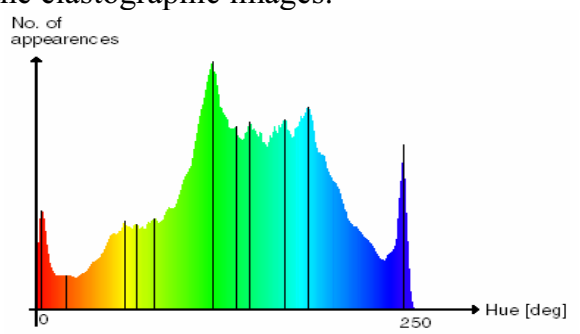

Figure 2. Filtered histogram for a region of interest in the elastographic image. The detected peaks are marked.

The problem of maximizing the log likelihood function can be solved using the expectation maximization algorithm proposed by Dempster et al for the incomplete data [5]. One of the problems associated with the expectation maximization algorithm is the initialization [6]. Both the accuracy of the solution and the number of iterations needed to find it are strongly dependent on the initial values of EM's parameters. This brings two kinds of disadvantages. The first disadvantage is that it is hard to avoid local maxima of the log-likelihood. The second is overfitting $[6,7]$. To overcome both these shortcomings the DAEM algorithm is used and, supplementary, a two step initialization is performed.

(1) Perform a histogram based detection of the peaks.

(2) Initialize the parameters fed to the DAEM algorithm with the values determined by the $\mathrm{k}$ means method [9].

We start by constructing a histogram of the hue values of the elastographic image or a region of interest in the image and seek to explicit peaks in the histogram. The motivation is that different classes of tissue in the image tend to give rise to peaks in the histogram. After smoothing the histogram, in order to 
eliminate perturbations causing an unnecessary number of maxima, these peaks are marked (figure 2).

Assume their number is $k$. Next the k-means algorithm is used for computing the parameters (mean, variance and prior probability) of each of the $k$ Gaussians. These values will be fed as initial values for the DAEM algorithm. This way the number of components is determined automatically. Furthermore, such an initialization yields a faster convergence and helps obtain better estimates [6].

The general DAEM approach has been thoroughly described in numerous papers. A comprehensive depiction of this algorithm adapted for image segmentation is found in [6,7 and 8].

This algorithm was adapted for automatic segmentation of the elastographic images, and for simplicity the same notations were used. First the initialization step is performed, as described above. Next the DAEM approach is tailored for elastographic image segmentation by employing a Gaussian Mixture Model (GMM) to represent the original distribution of the hue value $y_{i}$ observed from the $i$-th pixel $i=1, \ldots, \mathrm{N}$, where $\mathrm{N}$ is the number of pixels in the image.

In the expectation step, we perform the estimation of the unobserved $\mathrm{z}$ values (group indicator vectors), conditioned on the observation, using the values from the last maximization step. Since the initial values of the parameters are not guaranteed to be near the true ones (i.e., the posterior density is unreliable), the annealing parameter $\beta$ is added in order to weaken the effect of this posterior in the early stages of EM [6]. Next, in the maximization step of the $t+1$-th iteration the intent is to choose the value of the distribution parameters $\Theta$ that maximizes the expected log likelihood. This iterations are repeated for each increasing value $\beta<1$, until $\beta=1$. Each such iteration ends when the convergence criterion $\left(\Theta_{(t+1)}-\Theta_{(t)}<T\right)$ is satisfied. The final step of our proposed algorithm is to assign each pixel of the original image to the cluster or segment with maximum posterior probability according to the computed set of parameters $\Theta$.

\section{Experimental Results}

The performance of the proposed method was validated through experiments on both phantom models, where the position and dimension of the lesion was known a priori, and on patients which already had a clear diagnostic, established by other methods.

A total number of 50 video sequences were processed: 20 - simulations on phantom, 30 - real patient data. We also performed a variety tests using a set of 300 images from the Radiology Clinic of Cluj-
Napoca with 120 phantom images and 180 patient images.
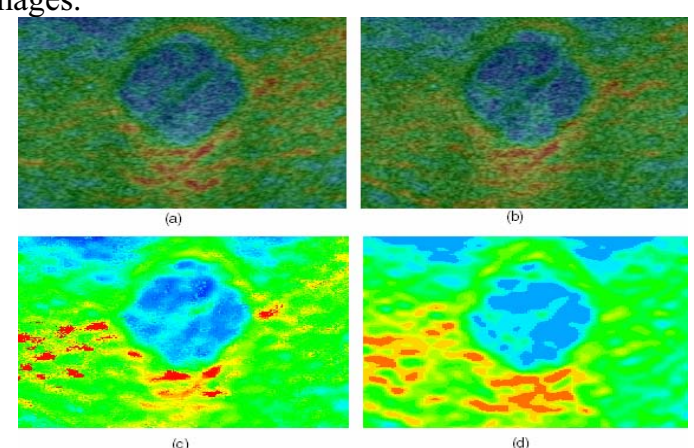

Figure 3. (a), (b) Elastographic information from phantom video sequence (2 frames out of 70); (c) Ultrasound image corresponding to (a); (c) Image with most probable values for each pixel, generated by processing the video sequence; (d) Result of our automatic segmentation method on image (a).
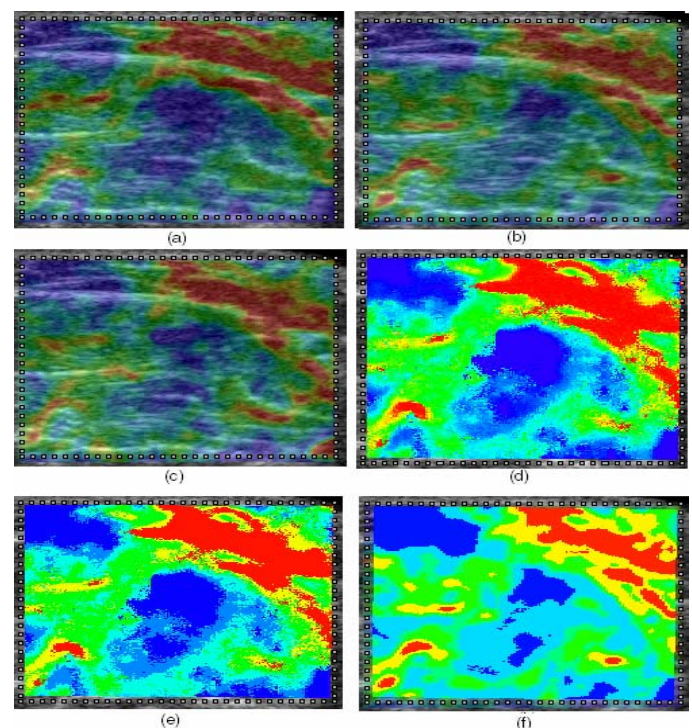

Figure 4. (a) - (c) Elastographic information form 3 frames extracted from a video sequence. Real patient data; (d) Image with most probable values for each pixel, generated by processing the video sequence from which (a)-(c) were extracted; (e) Automatic

segmentation algorithm performed on image in (d); (f) Automatic segmentation algorithm performed on image in (a).

By examining the phantom datasets an objective evaluation was performed, since the exact size and composition of each lesion was known prior. This way we validated both the proposed model for the most probable image and the segmentation procedure. By processing a 70 frame long video sequence (2 frames are shown in fig.3) the most probable image illustrated 
in figure $3 \mathrm{c}$ is obtained. In the case of phantom images, the area and the composition of the 'lesion' objectively validated the results obtained by our segmentation algorithm. The automatic segmentation of the image in fig. $3 \mathrm{a}$ is shown in fig. $3 \mathrm{~d}$.

The images generated as a result of video sequences processing visibly offer not only a better view of the underlying tissue but also offer a higher degree of certainty and values closer to the ground truth, as shown in figure 3 . The 20 lesion types in our phantom test images have been detected and segmented with an error of $5 \%$. Since, to our knowledge, no other segmentation algorithms have been proposed so far explicitly for elastographic images, there is no way of making a comparison with other methods. The same is true for the presented probabilistic model which generates the most probable data.

The examination of patient data subjectively validated our method. The diagnostic of the patient was clearly established prior to data acquisition by other methods. The type and shape of the identified lesion corroborated the prior diagnostic. Figure 4 shows 3 frames extracted from a 74 frames long video sequence together with the computed most probable image, the segmentation of the most probable image and the segmentation of a single frame.

Our proposed statistical models successfully provide not only an improved qualitative value, but also numerical quantification to the elastographic diagnostic method. On the most probable image, lesions can be numerically characterized by defining a region of interest and computing numerical features like average stiffness (average hue), standard deviation and other second order statistics that are defining for that lesion. For characterizing complex structures, the automatic segmentation algorithm can be used. A segmented image provides insight on the number of lesions and the area occupied by each lesion with respect to the area of the region of interest. This numerical information can be used to discriminate between lesions with different scores [10], which are ultimately of diagnostic value.

\section{Conclusions}

In this paper we proposed two statistical methods for adding diagnostic value to the elastographic images. The first method eliminates rough errors present due to the variability introduced by the operator using a "free hand" technique. The second, an unsupervised segmentation algorithm based on DAEM, offers accurate and fine segmentation of elastographic images by automatically detecting the number of clusters. The initialization method reduces the number of iterations needed for convergence and thus improves the execution time. We concluded from experiments on both phantom and patient data that the proposed algorithms been proven to perform well on determining the most probable data out of a video sequence and on accurately segmenting the elastographic image. Used together or independently, these statistical methods improve the quality of the diagnostic offered by elastography.

The fact that the number of clusters is determined automatically gives the method domain independence. This approach can be easily adapted and applied in any other domain where image segmentation is needed. This is done by simply changing the analyzed feature to another feature or to a feature vector.

\section{References}

[1] Ophir J., Cespedes I., Ponnekanti H., Yazdi Y., Li X. "Elastography: a quantitative method for imaging the elasticity of biological tissues." Ultrasonic Imaging 1991, $13,111-134$

[2] Jaros J. - Ultrasound Elastography - seminar LUT2, University of Kuopio, Finland -

venda.uku.fi/opiskelu/kurssit/LUT2/elastography.pdf

[3] S.M. Dudea, S. Nedevschi, C.D. Pantilie, C. Botar-Jid, D. Dumitriu, T. Marita, Ultrasound Elastography: from hysical Principles to Computer-Aided Image Analysis and Quantification,

[4] R. Chandrasekhar, J. Ophir, T. Krouskop and K. Ophir, Elastographic Image Quality vs. Tissue Motion in Vivo, Ultrasound in Medicine \& Biology, Vol. 32, No.6, pp. 847855, 2006

[5] A. P. Dempster, N. M. Laird and D. B. Rubin, Maximum Likelihood from Incomplete Data via the EM Algorithm, J. Roy. Stat. Soc. B, pp 1-38, 1977

[6] N. Ueda, R. Nakano, Deterministic Annealing EM Algorithm, Neural Networks, Vol. 11, Issue 2, pp 271-282, 1998

[7] W. H. Cho, S. Y. Park, J. H. Park, Segmentation of Color Image Using Deterministic Annealing EM, $15^{\text {th }}$ International Conference on Pattern Recognition, Barcelona, Vol. 3, pp 646-649, 2000

[8] T. Hoffman, J. M. Buhman, Pairwise Data Clustering by Deterministic Annealing, IEEE Transactions on PAMI, Vol. 19, pp 1-13, 1997

[9] J. B. MacQueen, Some Methods for Classification and Analysis of Multivariate Observations, Proceedings of 5-th Berkeley Symposium on Mathematical Statistics and Probability, Berkeley, University of California Press, 281297

[10] A. Itoh, E. Ueno, E. Tohno, H. Kamma, H. Takahashi, T. Shiina, M. Yamakawa, T. Matsumura, Breast Disease: Clinical Application of US Elastography for Diagnosis, Radiology, pp. 341-350, 2006 